\title{
Intestinal and hepatic fibrosis: how are they similar?
}

Geert D’Haens ${ }^{1}$, Massimo Pinzani²

1Amsterdam University Medical Centre, Academic Medical Centre, Department of Gastroenterology and Hepatology, Amsterdam, The Netherlands

2UCL Institute for Liver and Digestive Health, Division of Medicine, Royal Free Hospital, London, UK

\section{Address correspondence to:}

Prof Dr Geert D'Haens

Department of Gastroenterology

Amsterdam University Medical Centre

Academic Medical Centre

Meibergdreef 9

1100 DZ Amsterdam

The Netherlands

g.dhaens@amc.uva.nl

\section{Abbreviations used in this paper}

aSMA alpha smooth muscle actin

CD Crohn's disease

ECM extracellular matrix

HBV hepatitis B virus

HCV hepatitis $C$ virus

IOIBD International Organization for Inflammatory Bowel Disease

MRI magnetic resonance imaging

NASH non-alcoholic steatohepatitis

PRO patient reported outcome

\section{Conflicts of interest}

Geert D’Haens: Consultancy Abbvie, Ablynx, Allergan, Alphabiomics, Amakem, Amgen, AM Pharma, Arena Pharmaceuticals, Biogen, Bristol Meiers Squibb, Boehringer Ingelheim, Celgene/Receptos, Celltrion, Echo Pharmaceuticals, Eli Lilly, Engene, Ferring, DrFALK Pharma, Galapagos, Genentech/Roche, Gilead, Glaxo Smith Kline, Gossamerbio, 
Pfizer, Immunic, Johnson and Johnson, Kintai Therapeutics, Millenium/Takeda, Medtronics, Mitsubishi Pharma, Merck Sharp Dome, Mundipharma, Nextbiotics, Novonordisk, Otsuka, Pfizer/Hospira, Photopill, Prodigest, Prometheus laboratories/Nestle, Progenity, Protagonist, RedHill; Robarts Clinical Trials, Samsung Bioepis, Sandoz, Seres/Nestle, Setpoint, Shire, Takeda, Teva, Tigenix, Tillotts, Topivert, Versant and Vifor.

Massimo Pinzani: Siemens: Inventor and patent holder ELF test, Echosens: Speakers bureau; Promethera: SAB/Consultancy; Neurovive; Chemomab; Median Technology; UCB Cell Tech; Boehringer Ingelheim; Engitix Ltd; 3P-Sense Ltd; Hepatotargets.

\section{Authors Contributions}

Geert D'Haens and Massimo Pinzani wrote this manuscript based on their joint lecture at the European Crohn's and Colitis Organization (ECCO) annual meeting in Copenhagen, March 2019.

Fibrosis formation is a response to tissue damage and inflammation characterized by excessive production of extracellular matrix (ECM) by activated mesenchymal cells. It is a complication of many chronic diseases, and occurs in virtually all organs.

Accumulating evidence indicates that the pathogenic pathways of organ-associated fibrosis are often similar. This Comment addresses the similarities and differences between intestinal and hepatic fibrosis and strives to define common pathways that might lead to the development of effective therapies for both diseases.

An inception cohort study in the USA and Canada revealed that 3 years after the diagnosis of Crohn's disease (CD) in children and adolescents, 63/913 (7\%) had fibrostenotic complications. ${ }^{1}$ Since many of the patients with a perforating phenotype also had a fibrostenotic component, the total incidence of fibrostenotic complications approached $10 \%$, which is in agreement with data from a Danish cohort study $10 \%$ fibrostenosis at diagnosis and $17 \%$ after 7 years).

The picture is more complex for hepatic fibrosis. Many chronic liver diseases, including viral hepatitis, autoimmune diseases, alcoholic liver disease, and non-alcohol steatohepatitis (NASH) can cause hepatic fibrosis. Over several years, liver fibrosis can progress to cirrhosis, in which genetic and epigenetic factors as well as obesity and alcohol consumption play a role. Liver fibrosis and cirrhosis are associated with significant morbidity and mortality, and with an increased risk of hepatocellular carcinoma. Current, but probably undervalued, worldwide estimations show that 844 million people have CLDs, with a mortality rate of 2 million deaths per year. Early fibrosis can largely be reversed, but whether this can occur for cirrhosis is less clear.

An analysis of 48 bowel resection specimens showed that the fibrotic process in CD occurs mostly in the submucosa, which contains hyperplastic smooth muscle cells, and in the muscle layer, which is characterized by thickening and hyperplasia. ${ }^{2}$ 
The fibrogenic pattern in the liver shows more variability, related to the aetiology. In viral or autoimmune hepatitis, inflammation is the driver of 'portal to central' fibrosis, with early portal hypertension ${ }^{3}$. In metabolic liver disease (alcohol/drugs) the initial mechanism is oxidative stress, which causes (central lobular) fibrosis even in the absence of necrosis and inflammation. Biliary fibrosis is typically 'portal to portal' and activation of the bile acid receptor may play a pivotal part. Myofibroblasts, particularly those originating from hepatic stellate cells, are critical in the development of every type of liver fibrosis/cirrhosis. These cells undergo increased proliferation and contractility contributing to portal hypertension, and amplification of hepatitis ${ }^{4}$. They are activated by soluble factors secreted by virtually all resident liver cells and by a variety of cells involved in innate and acquired immunity.

Treatment of intestinal strictures is currently limited to balloon dilation, strictureplasty and surgical resections. Although effective this approach remains suboptimal. Following resection, the majority of $C D$ patients develop clinical recurrence and need further medical treatment and in about $20 \%$ a second resection is needed. Likewise, no antifibrotic agents have been registered for hepatic fibrosis. Although the eradication of HCV has been associated with an overall reduction in the incidence of hepatocellular carcinoma, the impact on hepatic fibrosis and cirrhosis has been disappointing. At present, only liver transplantation offers a solution for liver cirrhosis.

Nonetheless, a deeper understanding of the molecular mechanisms leading to intestinal and liver fibrogenesis has led to multiple drug development programs that may eventually lead to a medical rather than a surgical solution. For instance, the RhoA pathway is a major player in the transition of fibroblasts to activated myofibroblasts, the main producers of ECM components in the gut ${ }^{5}$. Blocking the RhoA pathway might therefore prevent/reverse intestinal fibrosis. In a recent preclinical study with antibodies against the receptor for interleukin 36 , a reduction of the severity of inflammation was demonstrated along with a decreased deposition of collagen, vimentin and alpha smooth muscle actin $(\alpha \mathrm{SMA})^{6}$. This molecule may not only prevent but also to reverse intestinal fibrosis. Another preclinical study showed that a topical inhibitor of ROCK decreased intestinal fibrosis and reduced expression of $\alpha$ SMA and metalloproteinase $9^{7}$. ROCK inhibitors however cannot be administered systemically given their significant cardiovascular toxicity. Other potentially antifibrotic agents for Crohn's disease include antibodies against TL1A and Oncostatin M.

In liver fibrosis, potential medical treatments have already reached phase 2 and 3 clinical trials. Interestingly, there has been surprisingly little overlap with agents studied for Crohn's disease. Obeticholic acid, targeting the farnesoid X receptor, was shown to have a significant anti-fibrotic effect without severe toxicity ${ }^{8}$. Cenicriviroc is a C-C chemokine receptor 2 and 5 antagonist with a significant anti-fibrotic effect at least during the first year of the trial ${ }^{9}$. A study with the promising galectin 3 antagonist GRMD-02 is ongoing. It is not unlikely that an effective drug for liver fibrosis will become available in the near future. 
To facilitate the evaluation of novel agents, 3D models have been generated by seeding cells from normal or cirrhotic human liver in scaffolds ${ }^{10}$. These models with human cells or plates on which ECM components are printed offer an attractive platform for drug development. Similarly, scaffolds were developed from decellularised human intestine subsequently recellularised with myofibroblasts or epithelial cells (M Pinzani, unpublished). This 3D model displays a natural intestinal structure with expression of key proteins allowing studying anti-fibrotic agents.

Whereas the transition from preclinical to clinical studies has already been somewhat successful in liver fibrosis (with endpoints still primarily based on liver histology, although non-invasive alternatives are being investigated), this next step has not yet taken place in Crohn's disease, despite the huge unmet need. Here, the major challenges include (1) effective delivery of the drug into hypovascularised fibrotic tissue, (2) the long duration of treatment that an antifibrotic intervention may require and (3) the need to target pathways of fibrosis and inflammation simultaneously. Furthermore, there is little consensus on the clinical trial endpoints to be used in interventional trials with anti-fibrotic drugs. In the uncontrolled CREOLE study an expert-developed 'obstructive symptom score' was used that showed a 64\% response rate to adalimumab. ${ }^{11}$ Most likely, clinical endpoints should be complemented by crosssectional imaging, such as ultrasound, CT and/or MRI. Recently, two consensus reports proposed potential endpoints for the study of anti-fibrotic agents in CD. ${ }^{12,13}$ Currently, the international Stenosis Therapy and Anti-fibrotic Research (STAR) consortium continues the development of these endpoints.

In conclusion, animal models indicate that intestinal and hepatic fibrosis may be reversible. Instruments for clinical trials, including imaging methods and patientreported outcomes are in development. The duration of treatments and potential toxicity are challenges. The future looks promising, but we need improved methodologies for target discovery.

\section{References}

1. Kugathasan S, Denson LA, Walters TD, et al. Prediction of complicated disease course for children newly diagnosed with Crohn's disease: a multicentre inception cohort study. Lancet 2017;389(10080):1710-18.

2. Chen W, Lu C, Hirota C, et al. Smooth muscle hyperplasia/hypertrophy is the most prominent histological change in Crohn's fibrostenosing bowel strictures: a semiquantitative analysis by using a novel histological grading scheme. I Crohns Colitis 2017;11(1):92-104.

3. Hall A, Germani G, Isgrò G, et al. Fibrosis distribution in explanted cirrhotic livers. Histopathology 2012;60(2):270-7.

4. Tsuchida T, and Friedman SL. Mechanisms of hepatic stellate cell activation. Nat Rev Gastroenterol Hepatol 2017;14(7):397-411. 
5. Johnson LA, Rodansky ES, Haak AJ, et al. Novel Rho/MRTF/SRF inhibitors block matrix-stiffness and TGF- $\beta$-induced fibrogenesis in human colonic myofibroblasts. Inflamm Bowel Dis 2014;20(1):154-65.

6. Scheibe K, Kersten C, Schmied A, et al. Inhibiting interleukin 36 receptor signaling reduces fibrosis in mice with chronic intestinal inflammation. Gastroenterology 2019;156(4):1082-97.

7. Holvoet T, Devriese S, Castermans K, et al. Treatment of intestinal fibrosis in experimental inflammatory bowel disease by the pleiotropic actions of a local Rho kinase inhibitor. Gastroenterology 2017;153(4):1054-67.

8. Younossi Z, Ratziu V, Loomba R, et al. Positive Results from REGENERATE: A Phase 3 International, Randomized, Placebo-Controlled Study Evaluating Obeticholic Acid Treatment for NASH. International Liver Congress 2019: abstract GS-06.

9. Friedman SL, Ratziu V, Harrison SA, et al. A randomized, placebo-controlled trial of cenicriviroc for treatment of nonalcoholic steatohepatitis with fibrosis. Hepatology 2018;67(5):1754-67.

10. Mazza G, Rombouts K, Rennie Hall A, et al. Decellularized human liver as a natural 3D-scaffold for liver bioengineering and transplantation. Sci Rep 2015;5:13079.

11. Bouhnik Y, Carbonnel F, Laharie D, et al. Efficacy of adalimumab in patients with Crohn's disease and symptomatic small bowel stricture: a multicentre, prospective, observational cohort (CREOLE) study. Gut 2018;67(1):53-60.

12. Rieder F, Bettenworth D, Ma C, et al. An expert consensus to standardise definitions, diagnosis and treatment targets for anti-fibrotic stricture therapies in Crohn's disease. Aliment Pharmacol Ther 2018;48:347-57.

13. Danese S, Bonovas S, Lopez A, et al. Identification of endpoints for development of antifibrosis drugs for treatment of Crohn's disease. Gastroenterology 2018;155:7687. 